\title{
LA $V$ EPISTEMOLÓGICA APLICADA A ALGUNOS ENFOQUES EN RESOLUCIÓN DE PROBLEMAS
}

ESCUDERO, CONSUELO ${ }^{1}$ y MOREIRA, MARCO ANTONIO ${ }^{2}$

${ }^{1}$ Departamento de Física. Facultad de Ingeniería. UNSJ. Av. Libertador, 1109 (oeste). 5400 San Juan. Argentina.

${ }^{2}$ Instituto de Física. UFRGS. Campus do Vale. Caixa Postal 15051. 91501-970 Porto Alegre. RS. Brasil.

\section{SUMMARY}

This paper includes a technique to apply a simplified Gowin's Vee as a reference tool to analyse four different trends which are useful for research in Physics problem solving. They are: $a$ ) the strategy alternative; $b$ ) the problem solving in accordance with scientific methodology; $c$ ) the problem solving and its social environment and $d$ ) the problem solving in Science and Technology with a practical proposal. Its analysis shows the way it can be used for the classroom.

\section{INTRODUCCIÓN}

La $V$ de Gowin ha mostrado ser un instrumento útil para el análisis del currículo, la evaluación y como recurso de enseñanza y aprendizaje (Novak y Gowin, 1988; Moreira, 1990b; Moreira y Buchweitz, 1993). Y también para el análisis del currículo de experimentos de laboratorio (Moreira y Levandowsky, 1983; Gurley-Dilger, 1992), de la estructura de una investigación (Moreira, 1990a) y del enunciado de un problema (Escudero, 1995).

La resolución de problemas, como muestra una abundante literatura (Costa y Moreira, 1995), es una de las líneas prioritarias de investigación en enseñanza de la física, tal vez, por la sencilla razón de que -así como hacer experiencias- resolver problemas es una actividad considerada indispensable para el aprendizaje de la física. Fundamentalmente, la investigación sobre resolución de problemas se ha orientado durante decenios a establecer diferencias entre buenos y mediocres «resolventes» $\mathrm{y}$ a diseñar algoritmos de resolución para facilitar la tarea de los alumnos.
En este trabajo examinamos el uso del diagrama $V$ desarrollado por Gowin (1981) como una herramienta útil para el análisis epistemológico de enfoques en resolución de problemas en física. Por análisis epistemológico vamos a entender el examen de interrelación entre el dominio conceptual (conceptos, principios, teorías...) y el dominio metodológico (registros, transformaciones, afirmaciones...), implícito en un modelo de resolución de problemas, a fin de producir conocimiento (p.e., la(s) respuesta(s) al problema). Las distintas orientaciones teóricas en investigación han generado distintos modelos normativos a ser eventualmente implementados en el aula o han interpretado la resolución de problemas, ya sea como una función cognitiva individual (Larkin y Reif, 1979; Mettes et al., 1980, 1981; Reif, 1981; Peduzzi, 1981; Mestre, 1991; Mestre y Touger, 1989) o como una actividad «repartida» $\mathrm{O}$ «distribuida» en la que el conocimiento es mutuamente construido por los participantes en dicha actividad (Nespor, 1990; Contreras, 1992). 
A continuación vamos a explicar someramente los elementos básicos de una $V$ epistemológica simplificada para ilustrar los elementos conceptuales y metodológicos que interactúan en un modelo de resolución de problemas y en la práctica habitual de resolución.

\section{LA $V$ DE GOWIN Y LA RESOLUCIÓN DE PROBLEMAS}

En 1977 Gowin inventó un heurístico en $V$ como medio para representar los elementos implicados en la estructura del conocimiento. En principio fue desarrollado para ayudar a clarificar la naturaleza y los objetivos del trabajo de laboratorio de ciencias (Novak y Gowin, 1988, p. 76) y, posteriormente, para descodificar el conocimiento «empaquetado» en artículos de investigación y su proceso de producción, en cualquier área.

Sin embargo, resolver un problema siguiendo un modelo explícito o implícito, en forma más o menos consciente o inconsciente, es una actividad cuya estructura también puede interpretarse en forma de una $V$. Los elementos básicos propuestos para esta $V$ simplificada son los que se muestran en la figura 1.

Figura 1

La $V$ epistemológica de Gowin simplificada.

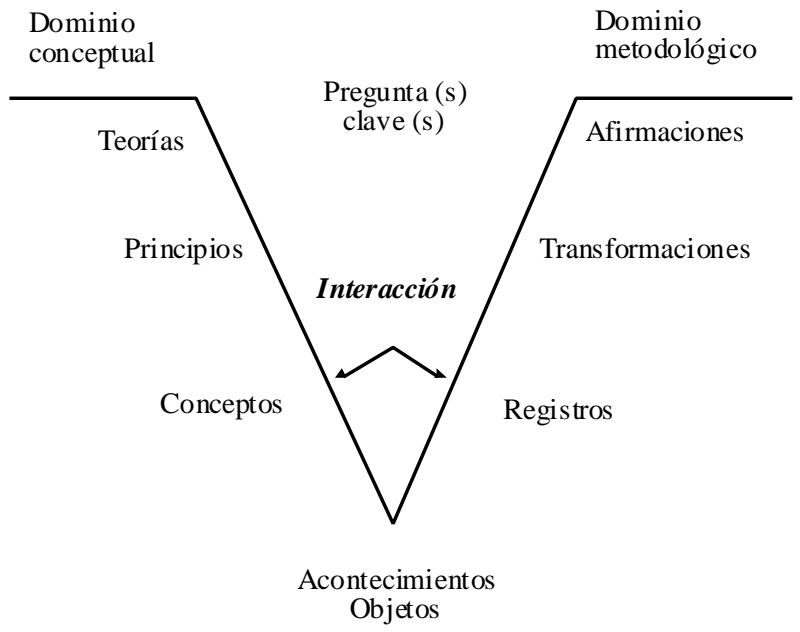

En la base de la $V$ se ubican los acontecimientos u objetos, fenómenos de interés sobre los cuales se formulan preguntas claves. Enlazados con estos acontecimientos están los conceptos y los registros de esos acontecimientos constituyendo la estructura más simple del conocimiento. Allí es donde se inicia la producción y creación del conocimiento. El lado izquierdo se refiere al aspecto conceptual de la producción de conocimiento (conceptos, constructos, principios, sistemas conceptuales, teorías, etc.), mientras el lado derecho se relaciona con los elementos metodológicos de esa producción (registros, datos, transformaciones, afirmaciones de conocimiento y de valor, etc.). El significado de nuestros registros siempre se interpreta a la luz del bagaje conceptual de que disponemos y, puesto que éste es limitado y evoluciona, sólo podemos hacer afirmaciones sobre cómo creemos que funciona el «trozo» de mundo que estamos estudiando.

Muchos de los modelos propuestos para la resolución de problemas insisten en tomar los datos como punto de partida de tal forma que todas las informaciones suministradas son necesarias y suficientes para que la situación esté completamente cerrada, lo que induciría según Gil Pérez y otros (1988a y b, 1992) a un tratamiento puramente operativo que lleva sólo a relacionar los datos, las incógnitas y las ecuaciones.

Por otro lado, desde la orientación sociocultural se han descrito cómo las actividades de resolución de problemas se manifiestan en el contexto social de la clase de física. Se puede examinar así la organización escolar como una actividad compartida por profesores y estudiantes que conduce a una comprensión común (Erickson,1982). Allí los profesores se refieren a los pasos generales normalmente seguidos al tratar un problema, comenzando con la extracción de datos. Además, en este caso específico, «resolver el problema» fue percibido como tomar la fórmula apropiada e «insertar» el dato «conocido». En ese enfoque hay evidencias de lo que puede llamarse la corriente lógica de la resolución de problemas en física y de que el fracaso no es del individuo sino del sistema sociocultural que «niega la oportunidad para el intercambio social y para un desarrollo cognitivo» (Contreras, 1992).

Ambas concepciones de la resolución de problemas, aunque desde posiciones diferentes, inducen a una discusión sobre cómo interpretar estos resultados y qué implicaciones podrían derivarse de ella para la práctica de la enseñanza y de la investigación.

Figura 2

Caso extremo de operativismo ciego en la resolución de problemas.

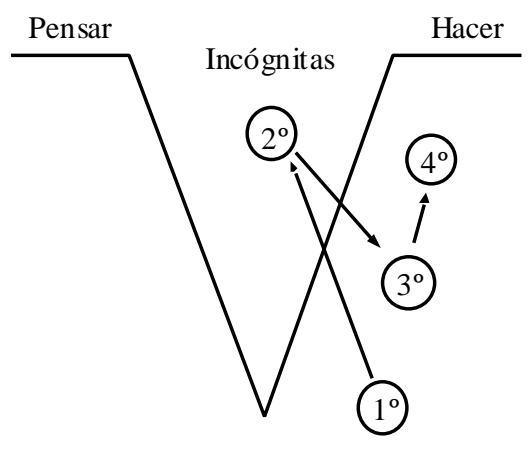


Si usamos la $V$ de Gowin como organizador (Fig. 2) para representar el trinomio datos, fórmulas e incógnitas, notamos que el problema se reduce a un sector de la misma. Se trata de un caso de operativismo ciego que frecuentemente denuncia la literatura en investigación. Éste pone en evidencia la ausencia de una integración (real) entre la teoría y la práctica, la que impide la «visualización» de los principales elementos que interactúan en la producción de conocimiento, imposibilitando un mayor análisis y acuerdo de significados.

A continuación aplicamos la $V$ de Gowin al análisis de cuatro propuestas de resolución de problemas con el propósito de clarificar en cierta forma sus visiones. Todas son amplias y llenas de matices, pero pretendemos principalmente analizar algunas de sus ideas en la práctica. No aspiramos a agotar dicha presentación.

\section{LA $V$ EPISTEMOLÓGICA Y ALGUNAS PROPUESTAS SOBRE LA RESOLUCION DE PROBLEMAS}

En esta parte del trabajo pretendemos realizar una aproximación a un análisis, reflexión y discusión de los elementos que interactúan en la resolución de un problema desde cuatro diferentes propuestas: $a$ ) la alternativa de una estrategia reformulada por Peduzzi; $b$ ) la resolución de problemas como investigación; $c$ ) la resolución de problemas y su contexto social; y d) la resolución de problemas en ciencias y tecnología como propuesta y presentación de resultados prácticos. Focalizaremos nuestra atención en algunos rasgos que nos permitan encontrar diferencias y semejanzas sobretodo relevantes para la práctica de la resolución de problemas en el aula. Creemos que los primeros problemas que se resuelven y los primeros momentos dedicados a su resolución son cruciales y definitorios en el sentido de ayudar a comprometer a nuestros alumnos en una resolución significativa.

Usamos la estructura de una $V$ de Gowin simplificada para señalar con números sucesivos la «secuencia»de resolución propuesta por un determinado modelo que ubicamos en el lugar de algún elemento de la $V$ con el cual notamos que se establece alguna correspondencia. Los números en las $V$ de cada modelo no se corresponden exactamente con el de la «secuencia» de resolución. A veces un mismo paso ocupa dos o más elementos en la $V$ y otras, dos o más etapas representan un sólo lugar en esta $V$ simplificada. También a veces el paso de un elemento a otro es simultáneo en más de una dirección. En otras palabras, los bordes no necesariamente resultan precisos entre lo que representa un paso o etapa de una secuencia y el lenguaje de la $V$, pero a pesar de ello nos permite arrojar luz acerca de lo que es un problema, sobre los elementos que intervienen en su solución y los aportes que nos pueden brindar las distintas orientaciones. Una vez aclarados estos detalles técnicos que nos pueden permitir una mejor interpretación de lo representado, comenzamos con el análisis de la estrategia propuesta por Peduzzi.

\section{LA RESOLUCIÓN DE PROBLEMAS EN FISICA: LA ATERNATIVA A UNA ESTRA- TEGIA}

La reformulación hecha por Peduzzi de un trabajo de Peduzzi y Moreira (1981) presenta y discute los ítems de una estrategia que tiende a contribuir a una mejor orientación del estudiante en relación con una situación problemática.

Dicho trabajo sugiere la siguiente estrategia para la resolución de problemas en física:

\section{Leer el problema con atención.}

II. Representar la situación-problema con diseños, gráficos o diagramas para su mejor visualización.

III. Extraer los datos (expresando las magnitudes involucradas en notación científica).

IV.Extraer la(s) magnitud(es) incógnita(s) expresándola(s) en notación simbólica.

V. Verificar si las unidades de las magnitudes involucradas forman parte de un mismo sistema de unidades; en caso negativo, estar atento a las transformaciones que sean necesarias.

VI. Analizar cualitativamente la situación-problema elaborando hipótesis si fuese necesario.

VII. Cuantificar la situación-problema, escribiendo una ecuación de definición, ley o principio en que está envuelta la incógnita y que sea adecuada al problema.

VIII. Situar y orientar el sistema de referencia a fin de facilitar la resolución del problema.

IX. Procurar desarrollar el problema literalmente haciendo las sustituciones numéricas sólo hacia el final o al final de cada etapa.

X. Analizar el resultado encontrado, verificando si es físicamente aceptable.

XI. Registrar en forma escrita los puntos «claves» en el proceso de solución.

XII. Cuestionar la situación problema.

En el lenguaje de la $V$ podríamos interpretar y traducir dicha estrategia como se muestra en la figura 3.

Se trata de una buena secuencia enriquecida con recomendaciones y sugerencias concretas producto de una gran capacidad y experiencia en el aula de los autores. Notamos un buen entrecruzamiento entre ambas ramas de la $V$, lo que redundaría en una mayor comprensión. $\mathrm{El}$ autor da, a nuestro entender, una excesiva importancia al tema de las unidades; no es que no lo tenga, sino que da la impresión de que lo prioriza, por ejemplo, frente al análisis cualitativo. Sobretodo si la transforma- 
ción de unidades es compleja, hacerla tan tempranamente puede favorecer que se pierda la idea física del problema y no se sepa como seguir, principalmente en aquellos alumnos con dificultades para resolver problemas.

\section{Figura 3}

La resolución de un problema de física a través de una estrategia sugerida por Peduzzi vista desde la $V$ de Gowin.

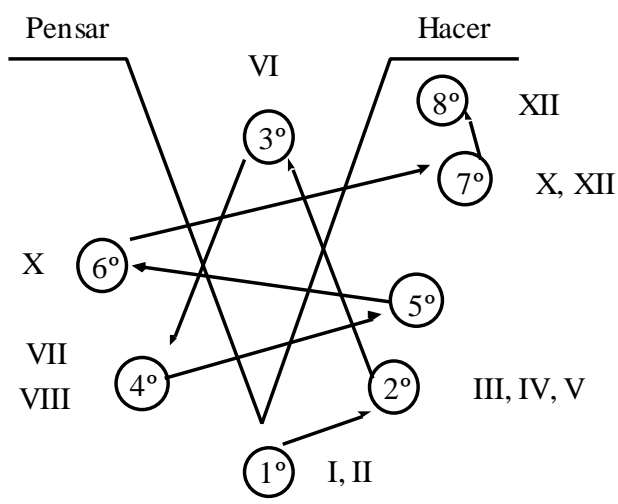

\section{LA RESOLUCIÓN DE PROBLEMAS COMO ACTIVIDAD DE INVESTIGACION}

El investigador español Gil Pérez en sucesivos trabajos viene cuestionando la didáctica de la resolución de problemas y defendiendo la utilidad y el potencial de uso de problemas no convencionales (de enunciado abierto). Una de las limitaciones que él entiende que ha tenido la investigación en resolución de problemas es que no se ha cuestionado en profundidad acerca de lo que se entiende por problema. Considera que un problema es una situación para la cual no se tiene una respuesta elaborada de entrada; por tanto, la solución se debería enfocar como una investigación científica. Una de las causas del fracaso generalizado de los estudiantes en la resolución de problemas según su perspectiva es culpa de la propia didáctica empleada. Los enunciados habituales dificultan un tratamiento científico de los problemas y dejan particularmente sin sentido la tarea fundamental de emisión de hipótesis.

Las orientaciones que sugiere que se proporcionen a los alumnos para abordar la resolución de problemas sin datos (en los que ya no es posible el simple juego de datos, fórmulas e incógnitas) serían básicamente las siguientes (Gil Pérez et al., 1988b, 1992):

I'. Considerar cuál puede ser el interés de la situación problemática abordada.

II'- I. Comenzar por un estudio cualitativo de la situación, intentando abordar y definir de manera precisa el problema, explicitando las condiciones que se consideren reinantes, etc.

III-II. Emitir hipótesis fundadas sobre los factores de los que puede depender la magnitud buscada y sobre la forma de esta dependencia, imaginando, en particular, casos límites de fácil interpretación física.

IV'- III. Elaborar y explicar posibles estrategias de resolución antes de proceder a ésta, evitando el puro ensayo y error. Buscar distintos modos de resolución para posibilitar la contrastación de los resultados obtenidos y mostrar la coherencia del cuerpo de conocimientos de que se dispone.

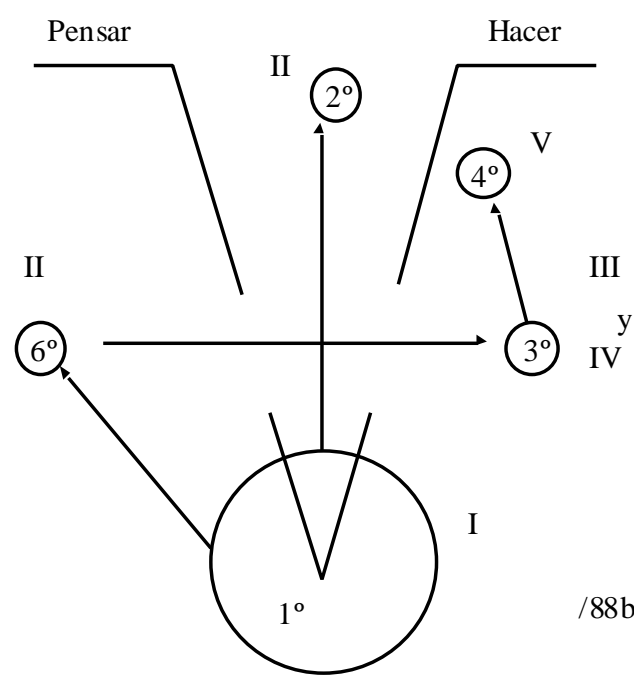

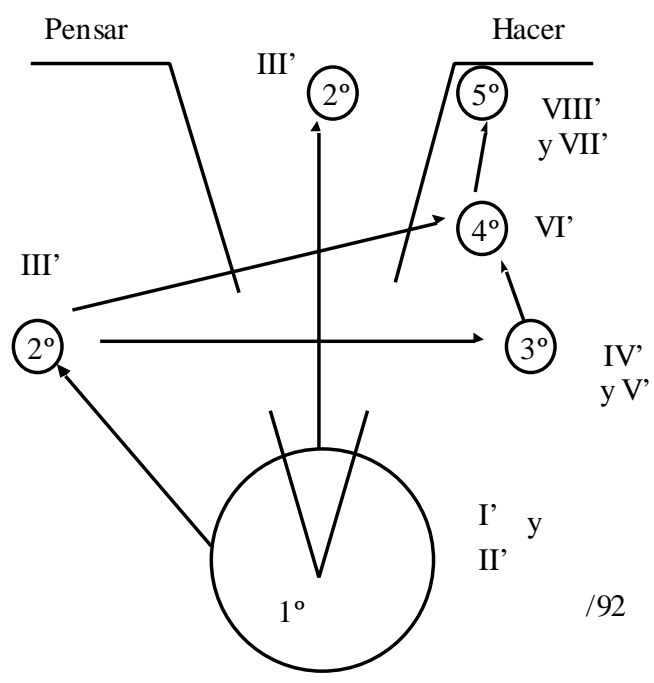

ENSEÑANZA DE LAS CIENCIAS, 1999, 17 (1) 
V'- IV. Realizar la resolución verbalizando al máximo, fundamentando lo que se hace y evitando, una vez más, operativismos carentes de significado físico.

VI' - V. Analizar cuidadosamente los resultados a la luz de las hipótesis elaboradas y, en particular, de los casos límites considerados.

VII'. Considerar las perspectivas abiertas por la investigación contemplando, por ejemplo, el interés de abordar la situación en un nivel de mayor complejidad o considerando sus implicaciones teóricas (profundización en la comprensión de algún concepto) o prácticas (posibilidades de aplicaciones técnicas). Concebir, particularmente, nuevas situaciones a investigar, sugeridas por el estudio realizado.

VIII'. Elaborar una memoria que explique el proceso de resolución y que destaque los aspectos de mayor interés en el tratamiento de la situación considerada.

Esta serie de orientaciones propuestas para facilitar el tratamiento de situaciones abiertas supone en conjunto un modelo de resolución de problemas como investigación (Gil y Martínez Torregrosa, 1983). Al analizar con la $V$ las dos versiones $(1988$ b, 1992$)$ de estas orientaciones obtenemos la figura 4.

La primera versión presenta cinco grandes orientaciones, que en la figura 4 marcamos en números romanos, mientras que en la versión más reciente señala ocho, que también indicamos en romanos pero «primados». Esta última incorpora aspectos como la consideración del interés de la situación problemática abordada (I), la de las perspectivas abiertas por la investigación realizada (VII') y también la elaboración de una memoria que explique el proceso de resolución y que incluya una reflexión global sobre lo que el trabajo realizado pudo haber abordado (VIII').

Los problemas abiertos requieren que se encuentre una expresión para la incógnita en función de los parámetros indicados por el propio solucionador; es decir, se tienen que imponer condiciones simplificadoras que dependen obviamente del cuerpo de conocimientos de que dispone el solucionador. En cambio, en los problemas cerrados el desarrollo literal es en función de los parámetros especificados por el enunciado.

Precisamente en los enunciados abiertos es el propio solucionador el que tiene que definir la situación que quiere resolver, decidir qué registros va a hacer y en función de qué conceptos. En la figura 4 hemos representado, con un círculo amplio que abarca acontecimientos, conceptos y registros, esta indiferenciación inicial que se irá definiendo en la medida que se vaya modelizando la situación. Si, por ejemplo, se tiene una situación que puede ser estudiada según distintos modelos, esto se tendrá que tener claro para poder tomar las decisiones del caso. Dicha elección va a determinar la «situación» en estudio, las condiciones que se consideren reinantes, los registros necesarios y las respuestas que se puedan dar. A nuestro parecer, es ahí donde se presenta la mayor dificultad a la hora de resolver problemas abiertos. En general, puede que no todos los alumnos estén preparados para enfrentarse a ellos o para poder resolverlos. Se necesita una buena dosis de entrenamiento y creatividad, entre otras cosas.

\section{LA RESOLUCIÓN DE PROBLEMAS Y SU CONTEXTO SOCIAL}

El trabajo de Contreras (1992) describe cómo profesores de física de secundaria construyen socialmente la resolución de problemas en sus interacciones diarias con los estudiantes y materiales curriculares.

Por un lado, presenta un marco alternativo para estudiar la resolución de problemas en su contexto natural contando con métodos de investigación interpretativos y sus constructos teóricos. Y en un sentido más práctico, este estudio etnográfico retrata la «clase de física» y más específicamente los acontecimientos de resolución de problemas como repartidos en contextos sociales en los que los estudiantes (y el profesor) están comprometidos «en hábitos alienantes de confianza en un repertorio de fórmulas» (Contreras, 1992) con los que realizan sus tareas diarias. Otro patrón común encontrado fue el de vincular a cada concepto emergente una fórmula individual que además se mostró independiente del curso en que se enseña y de la experiencia del profesor.

La figura 5 representa tanto la referencia hecha por un profesor acerca de los pasos o «fases» normalmente seguidos cuando se resuelven problemas numéricos como la «rutina» que sigue en el pizarrón al enseñar a resolverlos. Las palabras de un profesor extraídas de una viñeta fueron:

P: «Como es usual [...] nosotros primero leemos el problema [...] copiamos los datos [...] verificamos las unidades [...] si el sistema es MKS [...] hacemos las transformaciones (de unidades) y luego resolvemos el problema [...] ¿Puede pasar un voluntario? (op. cit., p. 22).

Además, los pasos se indican claramente por toda la transcripción de la clase y, luego de señalarlos, el profesor inicia la «solución» (dando las fórmulas de trabajo y fuerza en ese caso) ya que no hubo voluntarios.

En este trabajo se destaca la «naturaleza alienante de la resolución de problemas» (Contreras, 1992) en las clases estudiadas y sus consecuencias para el aprendizaje cognitivo. Aprender física consiste en copiar conceptos, leyes y principios relacionados con tópicos de esta disciplina y memorizar y archivar fórmulas asociadas a estos tópicos. Estas fórmulas eventualmente se convierten en el camino para pasar exámenes finales. En esta práctica, la comprensión conceptual es muy baja así como la metodológica, hasta tal punto que muchas veces son incapaces de encontrar la ecuación adecuada de una lista de fórmulas, porque confían en que al tenerlas se van a dar cuenta de cuál es la correcta. Este trabajo es importante entre otras cosas porque marca la necesidad, 
por un lado, de mirar profundamente hacia las condiciones que impiden a los estudiantes de ciencias a comprometerse en su propia construcción del conocimiento de la física y, por otro lado, de aprender más acerca del contexto inmediato de cognición de los estudiantes.

Figura 5

Referencia de los pasos o «fases» normalmente seguidos para resolver problemas en las clases de física de secundaria, según Contreras (1992), vista desde la $V$ de Gowin.

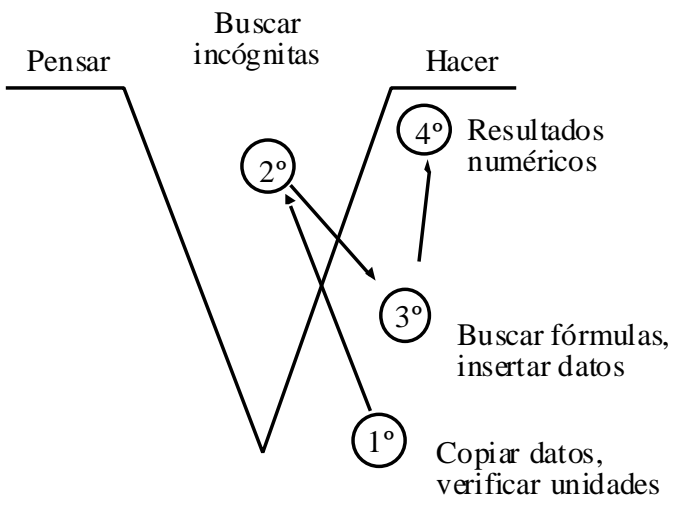

Desde esta perspectiva, la resolución de problemas es un «acontecimiento cognitivosocial» en el cual estudiantes y profesores construyen conocimiento escolar como conocimiento social (Nespor, 1990). Esto significa que resolver problemas (y aprender a hacerlos) no es sólo un proceso psicológico individual sino que es también una actividad interactiva reflexiva entre el estudiante y su ambiente inmediato (incluyendo profesores, compañeros y materiales curriculares) (Erickson, 1982). No obstante, dicha actividad debería involucrar también, de manera significativa, el dominio conceptual de los problemas, no sólo el metodológico (ni tan pobremente como lo sugiere la figura 5).

\section{LA RESOLUCIÓN DE PROBLEMAS EN CIENCIA Y TECNOLOGIA COMO PRO- PUESTA, Y PRESENTACIÓN DE RESULTA- DOS PRÁCTICOS}

En un trabajo reciente, Watts (1994) propone como modelo más usual de resolución de problemas una especie de proceso cíclico o en espiral que involucra:

I. Diseñar el problema. Eso significa establecer la forma usual del problema y cuáles son los mayores impedimentos u obstáculos para su inmediata solución.

II. Establecer criterios de éxito y los límites generales para su solución.

III. Generar ideas (torbellino de ideas) que tienen la finalidad de producir un dilatado rango de posibles soluciones.
IV. La elección de un conjunto limitado de posibles soluciones y la utilización de criterios para valuar recursos, materiales, conocimiento, tiempo, etc.

V. Uso del pensamiento y del desarrollo utilizando fuentes, expertos, recursos, etc. transferidos desde una franja de contextos y problemas previos; es decir, lo conocido.

VI. Ensayos iniciales y detección de las ideas más promisorias y un desarrollo racional de la solución más apropiada dadas las circunstancias.

VII. Construcción y prueba de prototipos que pueden ser modificados para llegar a una solución depurada.

VIII. Evaluación de los resultados o nuevo análisis de los criterios de éxito establecidos al comienzo.

En el lenguaje de la $V$ podríamos interpretar y traducir dicho modelo de resolución de problemas como se muestra en la figura 6 .

Si seguimos el proceso sugerido por Watts en la $V$, notamos que primero propone diseñar el problema para, después, establecer los criterios de éxito. Luego generar ideas sobre posibles soluciones, seleccionar un conjunto limitado de ellas y ensayar la solución más apropiada utilizando lo ya conocido. A continuación, construir y probar prototipos modificándolos hasta llegar a una solución depurada. Y, para finalizar, evaluar los resultados o analizar nuevamente los criterios de éxito que se establecieron en un principio.

Figura 6

Modelo más usual de resolución de problemas en ciencias y tecnología indicado por Watts (1994) y visualizado desde la $V$ de Gowin.

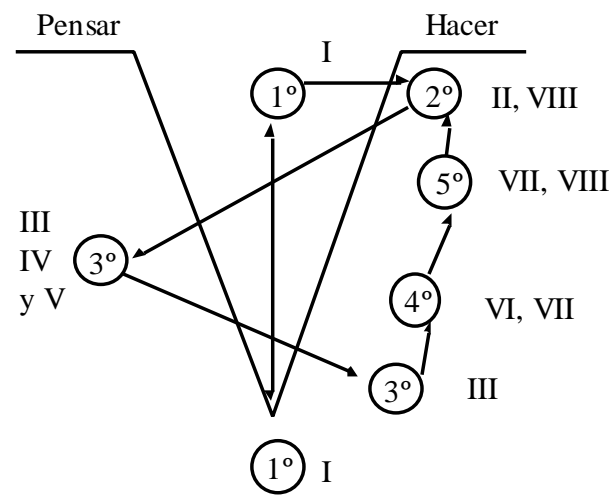

Para Watts, los problemas pueden ser experimentales o teóricos, con variados niveles de dificultad, cerrados o abiertos y con una o más soluciones. Los problemas de final abierto (Watts, 1994) comúnmente se caracterizan por: tener una o varias soluciones posibles; generar una 
variedad de resultados verosímiles y provechosos; envolver una amplia franja de estrategias y procesos; y atravesar la materia y los límites disciplinarios. En contraposición, los problemas cerrados se describen como aquéllos que conducen a una sola respuesta o, en todo caso, a un número de buenos resultados; tienen, generalmente, un resultado numérico o cuantitativo; envuelven una ruta prescriptiva para arribar al punto final; y pertenecen a una sola materia o disciplina.

Watts considera la resolución de problemas como una mediación que atraviesa el currículo y vincula un amplio rango de técnicas y procesos. Esto incluye una variedad de «caminos» y modos de aprendizaje que involucran conocimiento «procesable» en el que se engloba la red interna del pensamiento y de la acción. Se enfatiza así la modificación y el refinamiento de la solución a la luz de la experiencia. Este «hacer con las manos», al parecer, dirigiría la decisión del «resolvedor»y este movimiento es el que incrementaría la autoconfianza y satisfacción personal. Esto le da un ribete muy pragmático a la resolución de problemas, bastante diferente de aquél que considera problemas a los que figuran al final de un capítulo. En pocas palabras, este autor entiende la resolución de problemas como la resolución de situaciones prácticas más que el ensayo de ejercicios abstractos.

\section{REFERENCIAS BIBLIOGRÁFICAS}

AUSUBEL, D. P. (1976). Psicología educativa. México: Trillas.

CONTRERAS, A. (1992). Physics problem solving and its social context in secondary school. Documento presentado en la 65 Reunión Anual de la National Association for Research in Science Teaching, EEUU.

COSTA, S. C. y MOREIRA, M. A. (1995). Resolução de problemas Y: Diferenças entre novatos e especialistas. Memoris REF IX. Salta, Argentina, pp. 28-38.

ERICKSON,F. (1982). Taught cognitive learning in its inmediate enviroments: a neglected topic in the Anthropology of Education. Anthropology and Education Quartely, 13(2), pp. 149-180.

ESCUDERO, C. (1995). La resolución de problemas en física: herramienta para reorganizar significados. Caderno Catarinense de Ensino de Física, 12(2).

ESCUDERO, C. y GONZÁLEZ, S. (1996). Resolución de problemas en nivel medio: un cambio cognitivo y social. Investigaões em Ensino de Ciências, 2(2). Site: http.// www.if.ufrgs.br/ensino/revista.htm.

\section{CONCLUSIÓN}

En este trabajo se ha aplicado el diagrama $V$ como un instrumento para el análisis epistemológico de cuatro enfoques en la investigación de resolución de problemas. Se espera que sea útil para profesores e investigadores clarificar las diferencias entre las distintas perspectivas. La heterogeneidad de capacidades e intereses en los alumnos y la diversidad de énfasis curriculares muchas veces exige contar con variadas alternativas de trabajo. En los cuatro enfoques analizados, los autores muestran una interacción entre dos componentes: el del pensamiento (rama izquierda de la $V$ ) y el de la actuación (rama derecha); ambos necesarios para la comprensión de la naturaleza y la producción del conocimiento. Sin embargo, en los distintos enfoques, el tenor de la interacción es diferente.

Naturalmente la $V$ también puede ser utilizada en el aula para realizar un análisis epistemológico del enunciado de un problema (Escudero, 1995). Precisamente en esa dirección formulamos una propuesta de uso de una $V$ simplificada como herramienta que puede ayudar a reorganizar significados al reflexionar acerca de la esencia de un problema, de su resolución y de los elementos básicos que lo configuran. La misma se explicita en el artículo referido con dos ejemplos resueltos, uno para nivel medio y otro para universitario básico.

GIL PÉREZ, D. et al. (1988a). La resolución de problemas de lápiz y papel como actividad de investigación. Investigación en la Escuela, 6, pp. 3-20.

GIL PÉREZ, D., MARTÍNEZ-TORREGROSA, J. y SENENT, F. (1988b). El fracaso en la resolución de problemas de física: una investigación orientada por nuevos supuestos. Enseñanza de las Ciencias, 6(2), pp. 131-146.

GIL PÉREZ, D. et al. (1992). Questionando a didática de resolução de problemas: elaboração de un modelo alternativo. Caderno Catarinense de Ensino de Física, 9(1), pp. 7-19.

GOWIN, D.B. (1981). Educating. Ithaca, Nueva York: Cornell University Press. Trad. cast., 1985. Hacia una teoría de la educación. Argentina: Ediciones Aragón.

GURLEY-DILGER, L. (1992). Gowin's Vee. (Linking the lecture and the laboratory). The Science Teacher, 59(3), pp. 50-57.

LARKIN y REIF (1979). Understanding and teaching problemsolving in physics. European Journal of Science Education, 1(2), pp. 191-203. 
MESTRE, J. (1991). Learning and instruccion in pre-college physical science. Physics Today, septiembre, pp. 56-62.

MESTRE, J. y TOUGER, J. (1989). Cognitive research. What's in it for physics teachers? The Physics Teacher, septiembre, pp. 447-456.

METTES, C.T.C.W., PILOT, A., ROSSINK, J.H. y KRAMERSPALS, H. (1980a). Teaching and learning problem solving in science. Part 1: A general strategy. Journal of Chemical Education, 57, pp. 882-885.

METTES, C.T.C.W., PILOT, A., ROSSINK, J.H. y KRAMERSPALS, H. (1980b). Teaching and learning problem solving in science. Part 2: Learning problem solving in a thermodynamycs course. Journal of Chemical Education, 58, pp. 51-55.

MOREIRA, M.A. y BUCHWEITZ, B. (1993). Novas estratégias de ensino e aprendizagem - os mapas conceptuais e o Vé epistemológico. Lisboa: Plátano Ediçôes Técnicas.

MOREIRA, M.A. y LEVANDOWSKY, C.E. (1983). Diferentes abordagens ao ensini de laboratório. Porto Alegre, Br. Editora da Universidade.

MOREIRA, M.A. (1990a). Pesquisa em ensino: aspectos metodológicos e referenciales teóricos a luz do Vé epistemológico de Gowin. São Paulo: Editora Pedagógica e Universitaria.

MOREIRA, M.A. (1990b). O Vé epistemológico de Gowin como recurso instruccional no ensino de Ciéncias. Trabajo presentado en el III Congreso Internacional sobre la Didáctica de las Ciencias y las Matemáticas. Santiago de Compostela.

NESPOR, J. (1990). The Jackhammer: a case of study of undergraduate physics problem solving in its social setting. Internacional Journal of Qualitative Studies in Education, 3(2), pp. 139-155.

NOVAK, J.D. y GOWIN, D.B., 1988. Aprendiendo a aprender. Barcelona: Martínez Roca.

PEDUZZI, L.O.Q. Comunicación personal. Reformulación de la estrategia presentada y discutida, en Peduzzi, L.O.Q. y Moreira, M.A., 1981. Solução de problemas en física: un estado sobre o efeito de uma estratégia. Rev. Bras. Fis., 11(4), pp. 1067-1083.

REIF, F. (1981). Teaching problem solving. A scientific approach. The Physics Teacher, mayo, pp. 310-316.

WATTS, M. (1994). Problem solving in science and tecnology: extending good classroom practice. Londres: D. Fulton Publishers.

[Artículo recibido en diciembre de 1995 y aceptado en junio de 1998.] 